\title{
ON THE HIGH COMPOSITIONAL UNIFORMITY OF THICK GaAlAs LAYERS GROWN BY LIQUID PHASE ELECTROEPITAXY*
}

\author{
Z.R. ŻYTKIEWICZ AND S. MIOTKOWSKA \\ Institute of Physics, Polish Academy of Sciences \\ Al. Lotników 32/46, 02-668 Warszawa, Poland
}

\begin{abstract}
The results of electroepitaxial growth of thick GaAlAs layers on GaAs substrates are presented. It is experimentally proven that effective convective mixing of the solution volume results in the compositional uniformity of GaAlAs layers, even in spite of the high compositional non-uniformity of the material supplying the solutes ( $\mathrm{Al}, \mathrm{As}$ ) to the solution during the growth of the layers. For the first time this allowed us to grow uniform GaAlAs layers with thicknesses up to $200-300 \mu \mathrm{m}$ in a wide composition range from a small $(5 \mathrm{~g})$ amount of solution.
\end{abstract}

PACS numbers: 68.55.Df, 81.10.Dn

\section{Introduction}

Liquid phase electroepitaxy (LPEE) is the growth technique in which epitaxial growth is initiated and sustained by passing electric current through the interface between the substrate and the solution, while the temperature of the whole system is kept constant (see [1] for review). The unique feature of layers grown by LPEE is their large thickness and high compositional uniformity in the growth direction as compared to the layers grown by standard liquid phase epitaxy (LPE). However, for a fixed growth temperature $T_{\mathrm{gr}}$ and solution volume, the thickness of the layer grown is limited by solubility of the elements (Al, As) in the solution. Therefore, a new version of LPEE - the modified LPEE - has been proposed $[2,3]$ in which the $\mathrm{Ga}-\mathrm{Al}-\mathrm{As}$ solution is saturated with $\mathrm{Al}$ and As at the temperature $T_{\mathrm{s}}>T_{\mathrm{gr}}$ and then cooled to $T_{\mathrm{gr}}$ in the absence of substrate (Fig. 1a). In this procedure the source GaAlAs layer is deposited on the excess GaAs wafer floating on the solution by the same mechanism which is responsible for epilayer growth during LPE. After the substrate was slided under the solution and the dc current was applied the GaAlAs source material was transported through the solution to the substrate and deposited as an electroenitaxial layer (Fig. 1a). As the

*This work is supported in part by the Committee for Scientific Research grant no. 204769101. 


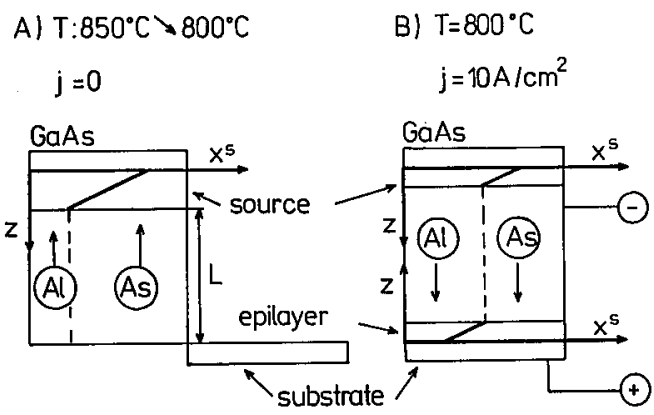

Fig. 1. Schematic drawing of the growth cell used in LPEE growth of GaAlAs. a) the source formation process, b) electroepitaxial growth on the substrate. The Al concentration $x^{\mathrm{s}}$ profiles in the source material and the electroepitaxial layer (if the simple model of direct source recrystallization is valid) are drawn by bold lines.

source material is grown by cooling, the change of temperature and the melt depletion should sharply grade its composition profile leading to a decrease of $\mathrm{Al}$ content in the solid with an increase of its thickness (Fig. 1b) - a phenomenon typical for LPE grown GaAlAs [4]. Therefore, the composition profile of the electroepitaxial layer, grown by recrystallization of the source material, should be graded too, but with the opposite sign of the composition gradient (Fig. 1b). Thus, the growth of layers with increasing $\mathrm{Al}$ content towards the surface is expected in our case. This is in contradiction to our previous results [3], where the same technique was used to obtain layers with a highly uniform composition profile. The explanation of the above discrepancy is the purpose of this paper.

\section{The growth procedure and experimental results}

The $\mathrm{Ga}_{1-x} \mathrm{Al}_{x}$ As epilayers were grown on (100) GaAs substrates at $T_{\mathrm{gr}}=$ $800^{\circ} \mathrm{C}$ with the electric current density $j=10 \mathrm{~A} / \mathrm{cm}^{2}$. Before the growth process the solution was saturated with $\mathrm{Al}$ and $\mathrm{As}$ at $T_{\mathrm{s}}=850^{\circ} \mathrm{C}$ and then cooled down to $T_{\mathrm{gr}}$ with the cooling rate of $0.1^{\circ} \mathrm{C} / \mathrm{min}$ to prepare the source material as discussed earlier. All the others details of the growth procedure can be found in [3]. The compositions of grown layers were determined by scanning electron microscope (SEM).

Figure 2 presents the composition profiles of layers grown from solutions of the same composition as a function of the amount of solution used for crystallization. It appears that depending on the thickness $L$ of the solution used we can obtain highly uniform epilayers as well as layers with $\mathrm{Al}$ content increasing with thickness. Changing the thickness of the solution we can control the contribution of convection to the solutes transport in liquid. Thus the results presented in Fig. 2 can be explained if the contribution of convection in melt is included. In the limit of a thin layer of solution there should be no convection and the solutes are transported towards the substrate by diffusion and electromigration. Then the picture of direct source material recrystallization (Fig. 1b) is valid and the layer 


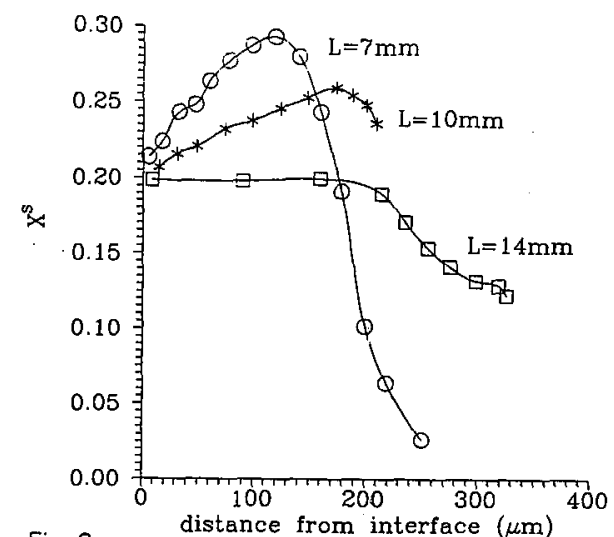

Fig. 2

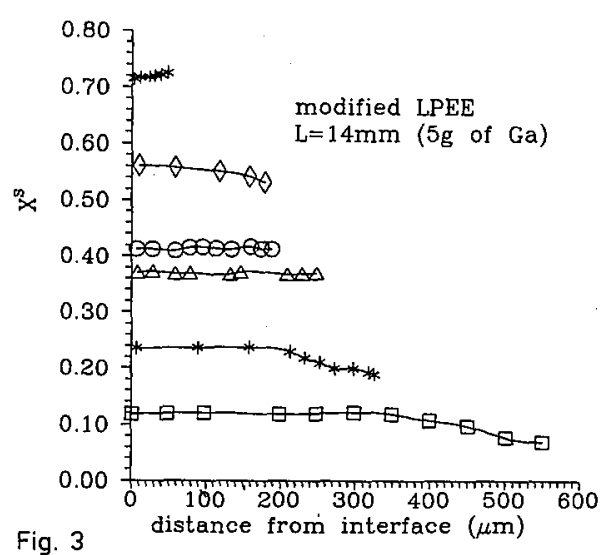

Fig. 3

Fig. 2. The composition profiles of layers grown from solutions of different thickness $L$. Fig. 3. The composition profiles of layers grown by modified LPEE from $14 \mathrm{~mm}$ thick solutions $(5 \mathrm{~g}$ of $\mathrm{Ga})$.

with increasing $\mathrm{Al}$ content is grown. When a thick layer of solution is used the contribution of convection cannot be neglected and two effects are expected. The presence of convection during the source preparation procedure should lead to a decrease of the composition gradient in the source [4], what in turn is propitious for the growth of uniform ternary layers. Moreover, the convective mixing of soIution volume breaks down the direct correspondence between the composition profiles of the source and epilayer, resulting in a smoothing of $\mathrm{Al}$ distribution in the grown GaAlAs. Thus, using $14 \mathrm{~mm}$ thick solutions (which corresponds to $5 \mathrm{~g}$ of $\mathrm{Ga}$ ) we were able to grow thick and homogeneous GaAlAs layers, despite the compositional non-uniformity of the source material.

Figure 3 presents the compositional profiles of layers grown by this method in a wide composition range. In our opinion, the difficulties with the successful growth of thicker layers with higher Al concentration are closely related to the problem of solution oxidation during growth.

\section{Conclusions}

We have shown the positive influence of convection in the solution on the compositional uniformity of GaAlAs layers grown by modified LPEE. Depending on the absence or presence of convection (i.e. on the thickness of the solution) layers with increasing $\mathrm{Al}$ content or uniform in composition, respectively, can be grown from the solution of the same composition. The growth of GaAlAs layers with both these types of composition profiles is very difficult by any other method of solution growth. 


\section{References}

[1] T. Bryśkiewicz, Prog. Cryst. Growth Charact. 12, 29 (1986).

[2] J.J. Daniele, A.J. Heblig, J. Appl. Phys. 52, 4325 (1981).

[3] Z.R. Żytkiewicz, S. Miotkowska, J. Cryst. Growth 121, 457 (1992).

[4] I. Crossley, M.B. Small, J. Cryst. Growth 15, 268 (1972). 